\title{
Efficient Rate-Power Allocation for OFDM in a Realistic Fading Environment
}

\author{
Kamau Prince, Brian Krongold and Subhrakanti Dey \\ ARC Special Research Centre for Ultra-Broadband Information Networks \\ Affiliated Program of National ICT Australia \\ University of Melbourne, Australia \\ Email: k.prince@ee.unimelb.edu.au,bsk@unimelb.edu.au, s.dey@ee.unimelb.edu.au
}

\begin{abstract}
The implementation of practical adaptive resource allocation scheme remains a key criterion to be satisfied for realising spectrally efficient multitone wireless communications. The ever-increasing demand for spectrally efficient broadband wireless transmission technologies has spurred intensive research leading towards the implementation of adaptive OFDM and adaptive MIMO systems. Efforts in this direction have been frustrated however by the lack of a clear and accurate description of the fading behaviour typically encountered in the broadband wireless transmission environment. This has been partially been overcome by the use of mathematical modelling which captures certain large-scale characteristics of the channel and facilitates theoretical research. The "average" channel parameters gleaned from these processes is typically then used to inform the design and configuration of wireless networking equipment after the broad application of generous safety margins. The resulting solution is therefore quite robust to certain transient channel quality degradation yet the generous safety tolerances render it unable to exploit other transient transmission quality improvements

We seek to overcome the problems associated with this approach by applying a theoretically sound novel adaptive resource allocation framework to actual broadband wireless channel development data. The allocation framework is derived from the optimal OFDM allocation scheme for a known channel [1]: the channel development data is obtained from actual measurement of a broadband wireless mobile environment [2]. Prediction techniques are employed to overcome the time lag between channel assessment and symbol transmission. We present the details of the predictive resource allocation scheme used and include a broad characterisation of the transmission environment in terms of the time-varying fading processes observed. We provide some results of the application of this scheme as typical performance levels that may be achieved in an actual transmission environment.
\end{abstract}

\section{INTRODUCTION}

Practical adaptive resource allocation has been remained an elusive target for the achievement of spectrally-efficient wireless communications. An important characteristic of such communications schemes is their ability to exploit transient channel behaviours, partly through increasing the utilisation of advantageous channels and avoiding severely degraded facilities: doing this could potentially maximise the overall transmission quality while minimising power consumption, for example. Rapid variations in the quality of the wireless channels used by such networks have however traditionally frustrated efforts to develop such an ideal adaptive resource allocation scheme. This is because the time lag typically associated with assessing the current channel state information
(CSI) and the determination of an optimised loading schedule is significant when compared to the timescale of the fading processes that influence channel quality variations, leading to the obsolescence of this schedule by the time it has been found.

A potential means of overcoming this problem is in the implementation of CSI prediction to determine the channel quality at a (future) transmission instant and optimising transmission resource distribution in time to send the desired data symbol. To this end, heavy interest has recently been invested in CSI prediction for systems employing OFDM transmissions [3]-[5] as well as in the integration of CSI prediction and adaptive resource allocation [6]-[8] in attempts to achieve the desired solution for this problem. While these approaches have yielded much success, it was observed that much of this work has been performed on analytical channel models, while in only a few examples such as [7] is actual channel data used. We seek to extend on this body of work by applying a novel resource allocation scheme to actual channel fading data and gain insight into typical results that can be obtained in similar real-world applications.

We demonstrate the application of our previously developed resource allocation scheme [8]-[10] to wireless channel fading as exhibited by an actual channnel. The data provided for this analysis was obtained as result of an evaluation performed of mobile wireless transmission in two locations in Stockholm, and was kindly made available to us by Ericsson Radio Systems AB: this data is from the same dataset used by [2]. LMS filtration [11] is applied to predict the CSI at the future instant of symbol transmission: this prediction horizon will be exploited to perform the transmission optimisation. We indicate how the channel fading processes influence the accuracy and range of this prediction.

In the next section, we characterise the channel fading dataset used as best as we can without violating the confidentiality agreement under which this data was provided. We briefly outline the operation of the prediction-based adaptive resource allocation framework used and its application in a realistic fading environment. Results will then be presented illustrating the successful operation of the resource allocation scheme in this environment. We conclude this paper with an analysis of these results and a discussion of the implications that they hold for practical dynamic resource allocation in a real-world fading environment. 


\section{Channel Development}

\section{A. Source Data}

The transmission channel data used was obtained by Ericsson Radio Systems AB of Stockholm, Sweden and was initially described in [2]. The first set of measurements was taken in Kista, an environment that may be described as having a mix of urban and suburban characteristics; the second set was captured in central Stockholm, an urban environment with areas of open water. In all cases, the base stations were 2-3 m above rooftop level, which was in turn always 20-25 m above ground level.

A mobile antenna was placed on top of a vehicle driven around local streets: most of the measurements were taken in non-line-of-sight conditions. A BPSK-modulated 511-bit PN sequence having a $3 \mathrm{~dB}$ bandwidth of slightly below $4.672 \mathrm{MHz}$ was utilised to assess the impulse response of the channel. The receiver was implemented using a HP 89441A Vector Signal Analyzer set to a sampling rate of $6.4 \mathrm{MHz}$. Each measurement was obtained as a set of 1430 complex response bins by cross correlation with the transmitted sequence. Vehicle speed was varied between $30-90 \mathrm{~km} / \mathrm{h}$ and the centre frequency of the transmission was $1880 \mathrm{MHz}$.

Post-processing was done on the measured impulse responses to $1.25 \mathrm{MHz}$ and $5 \mathrm{MHz}$ bandwidths and sampled at rates of 1.28 these bandwidths to avoid aliasing in the receiver. The median noise level was also assessed and all received samples less than $6 d B$ above this level were set to zero in a manner similar to [12]. Additional details of the data acquisition process are presented in [2].

We selected a group of twenty channel datasets and evaluated the 512-point discrete Fourier transform (DFT) of the measured data in order to determine the frequency response of the wireless channel and hence the associated power spectral density (PSD) of the fading observation. Fig. 1 indicates the range traversed by the PSD within the cutoff bandwidth. The mean gain value of the transmission channel is also indicated on this diagram. The unequal gain observed across the frequency range of interest indicates the possible existence of frequency-selective fading phenomena.

We chose one continuous realisation of the fading process from these twenty sets of data to represent the conditions under which further analysis take place. We evaluated the real and imaginary parts of the autocorrelation function of the channel gain observations, and present these in Fig. 2 and Fig. 3 respectively. We also present the autocorrelation of the absolute magnitude of the fading realisations as Fig. 4. We observe from Fig. 4 that the autocorrelation of the fade magnitude remains greater than zero for a lag of $-1000 \geq$ $\tau \geq 1000$, which indicates that CSI prediction is possible on this source data within these bounds (at least).

\section{B. System Model}

Without loss of generality, we are interested in either the uplink or downlink channel between transceivers in a mobile wireless environment. We assume that a means exists for

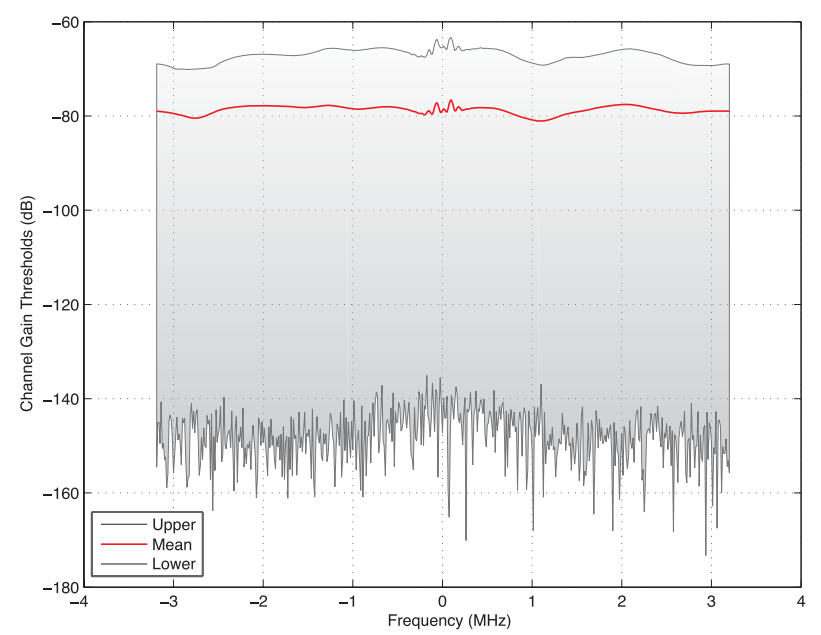

Figure 1: Range of PSD of Ericsson Channel

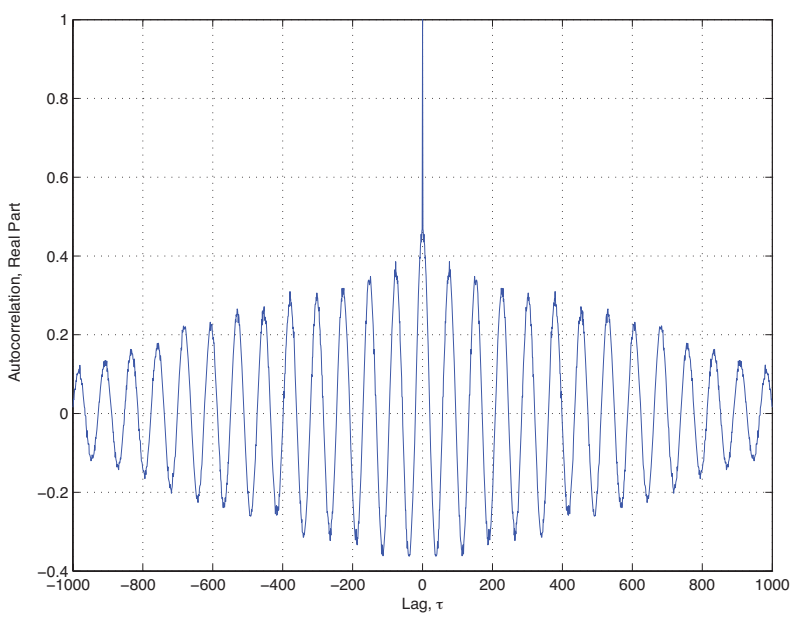

Figure 2: Real Part of Fading Autocorrelation

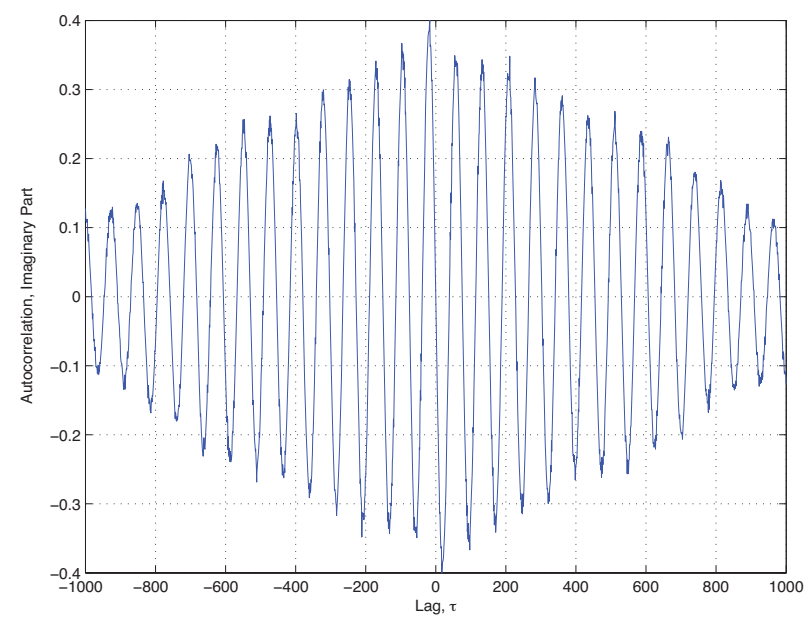

Figure 3: Imaginary Part of Fading Autocorrelation 


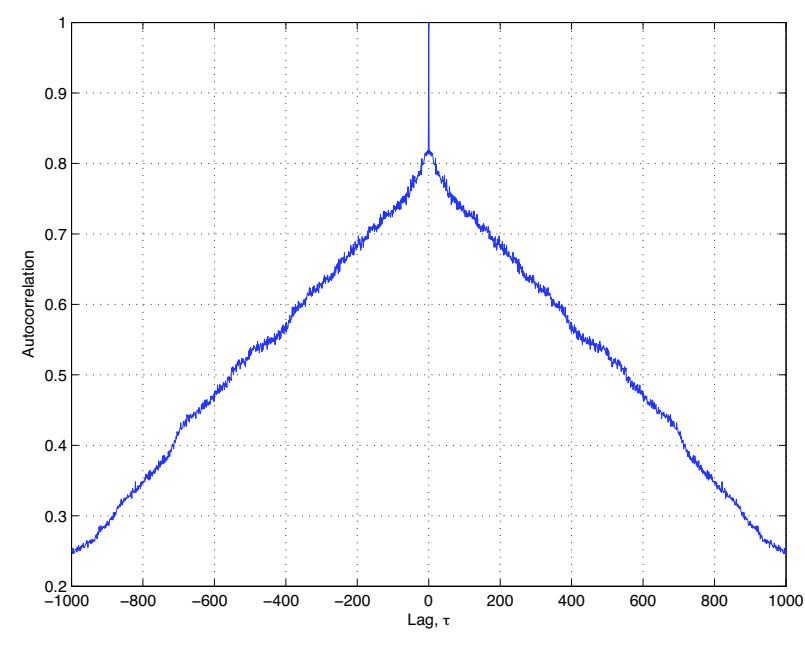

Figure 4: Autorrelation of Fade Magnitude

accurately estimating the prevailing CSI and reliably relaying this information between transceivers in a reasonable amount of time, thus facilitating adaptive resource allocation. We consider the transmission system of interest as operating on discrete time intervals $k \in\{1,2, \ldots, 1430\}$, in the presence of a wide-band channel divided into $N$ narrowband subcarriers. We also consider that the (nontrivial) channel fading attenuates a given transmitted symbol (denoted $\mathbf{x}(k) \in \mathbb{C}^{N}$ ) such that the power received on each subcarrier $i \in \mathcal{I}=\{1, \ldots, N\}$ has been modulated by an amount $\alpha_{i}(k)$ : in this way, we characterise the effect of channel fading on received symbol power by the (real) vector $\boldsymbol{\alpha}_{k}=\left[\alpha_{i}(k)\right]_{\forall i \in \mathcal{I}}$. No loss in generality is incurred by characterising $\boldsymbol{\alpha}(k) \in \mathbb{R}^{N}$ as OFDM transmissions implement a cyclic prefix that may be exploited at the receiver to eliminate any phase offset effects incurred during transmission.

\section{CSI Prediction}

We sought to implement CSI prediction on $N=6$ uniformly-spaced subchannel frequencies within the available bandwidth. We assumed an autoregressive channel model and implemented LMS predictive filtering [11] of the DFT estimate of the subchannel gain after the application of an appropriate normalizing factor. Let $d \geq 1$ denote the prediction horizon of the filter, $F$ the filter order and $\mathbf{w}_{k} \in \mathbb{C}^{F}$ be the filter weight values. We utilise the set of observed values $\mathbf{u}(k)$ such that

$$
\left(\mathbf{u}_{k}\right)^{\mathrm{T}}=\left[\boldsymbol{\alpha}_{k} \boldsymbol{\alpha}_{k-1} \ldots \boldsymbol{\alpha}_{k-F}\right]
$$

to determine $\boldsymbol{y}_{k+d}=\left[y_{i}(k+d)\right]_{\forall i \in \mathcal{I}}$ the LMS estimate of the observed fading at the interval of interest:

$$
\begin{aligned}
y_{i}(k+d) & =\mathbf{w}_{k}^{\mathrm{T}} \times \mathbf{u}_{k} \\
\mathbf{h}_{k} & =\mathbf{u}_{k} \times\left(\boldsymbol{\alpha}_{k}-\mathbf{y}_{k+d}\right)^{\mathrm{H}} \\
\mathbf{w}_{k+1} & =\mathbf{w}_{k}+\mu \times \mathbf{h}_{k}
\end{aligned}
$$

A normalisation factor of $10^{4}$ was applied to the fading data, and an analysis of the predictor mean square error (MSE)

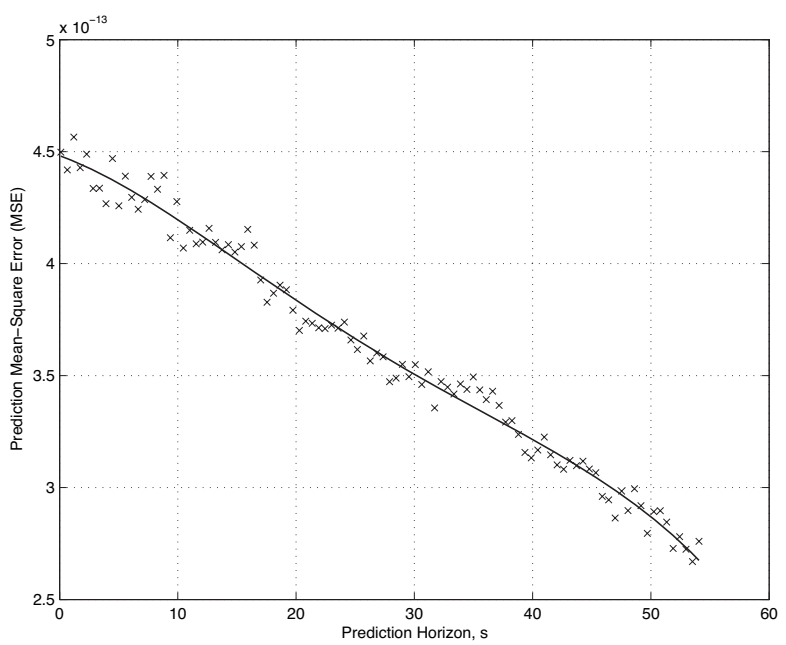

Figure 5: Variation of Prediction MSE with Prediction Depth

performance is presented as Fig. 5 for a step-size value of $\mu=0.1$ and a filter order $F=2$. A $4^{\text {th }}$ order polynomial line of best fit is also displayed. We observe that the prediction MSE starts from an approximate maximum value of $4.5 \times$ $10^{-13}$ for $d=1$ and then diminishes to below $2.9 \times 10^{-13}$ as the prediction horizon is increased to 50 seconds. We also note that the MSE error performance is negligible compared to the average gain thresholds identified in Fig. 1: this indicates that accurate LMS-based CSI prediction is possible within this fading environment.

We also observe that the MSE of the prediction is significant when compared to the minimum PSD of the fading phenomenon. This indicates the possibility that the gain realised on a given subchannel $\alpha_{i}(k)<y_{i}(k)$, and hence that data transmistted over the channel at this time will be received a lower SNR than required to satisfy transmission quality of service (QoS) requirements. We denote such an event as a shortfall, and that such a condition would lead to a transmission outage on that subchannel at that time. We note that a possible means of mitigating for these effects would be to scale the predicted $\mathbf{y}_{k}$ by a suitable margin $\varphi$ prior to performing resource allocation.

In investigating the efficacy of such a scaling scheme, we examine the effect of scaling the predicted $\mathbf{y}_{k}$ by various amounts and observing the predictor shortfall performance, $\mathcal{P}_{\mathrm{S}}(\varphi, d)$, as defined below:

$$
\mathcal{P}_{\mathrm{S}}(\varphi, d):=\mathbb{P}\left[\alpha_{i}(k)<\varphi \cdot y_{i}(k), \forall i \in \mathcal{I}, 1 \geq k \geq 1430\right]
$$

In further discussion, we will denote the shortfall probability by a simplified $\mathcal{P}_{\mathrm{s}}$. We evaluated the effect of various scaling factors, $0.8 \geq \varphi \geq 1$ with $\mu=0.1$ and $F=2$ and present the results as Fig. 6. A $4^{\text {th }}$ order polynomial line of best fit for each set of data is also presented. These results indicate that a decreasing value of $\mathcal{P}_{\mathrm{S}}$ is observed with an increasing prediction horizon is increased, for a fixed $\varphi$. This trend that is similar to the one observed in Fig. 5. Reducing $\varphi$ results in a reduction in $\mathcal{P}_{\mathrm{S}}$ at a given prediction horizon. 


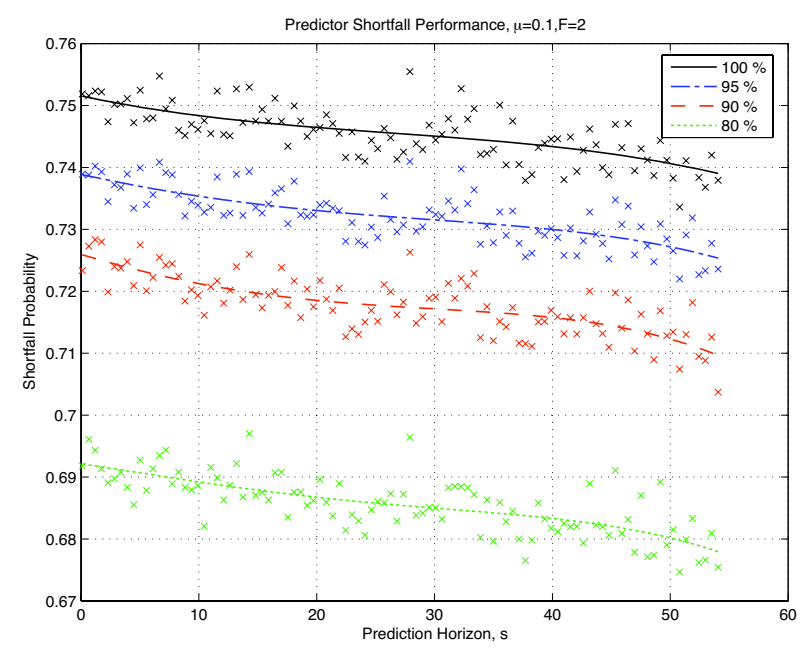

Figure 6: Variation of Shortfall Error vs. Prediction Depth

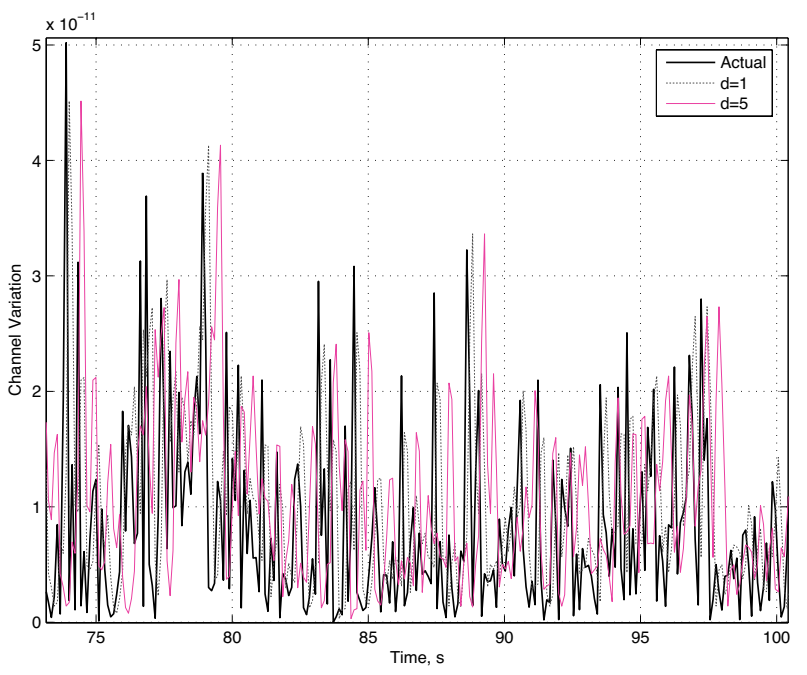

Figure 7: Prediction of Single Channel Gain

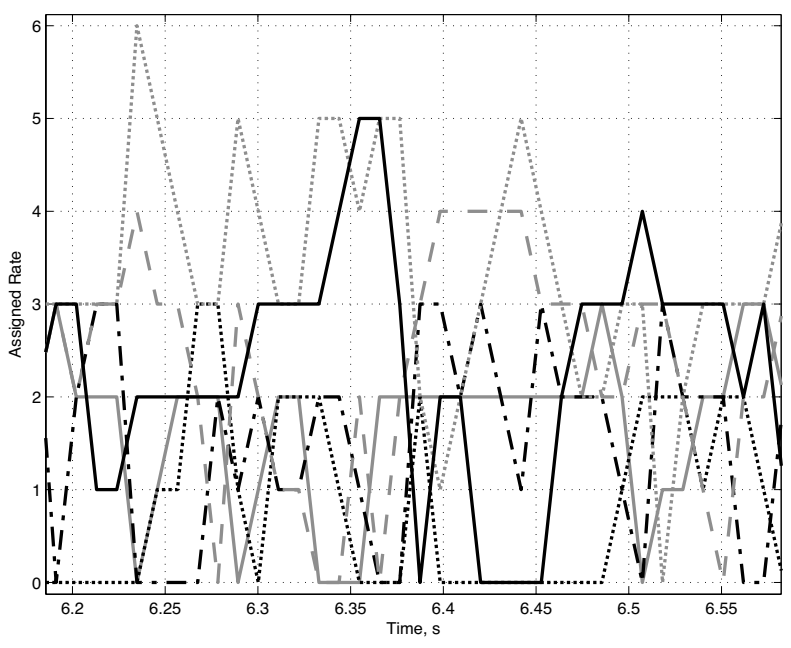

Figure 8: Variation of Subchannel Rate over Time

\section{Resource Allocation}

The results of the previous section indicate that it is feasible to perform CSI prediction in the presence of actual channel fading. Additionally, the strong correlation observed in channel fading at low lag, in addition to the low complexity of the LMS predictor makes such a method viable for performing adaptive resource allocation. We thus investigate the application of CSI prediction in conjunction with adaptive resource allocation to the real-world situation.

Following [9], [10], we apply an optimal resource allocation scheme for the known OFDM channel [13] to the predicted CSI value $\hat{\boldsymbol{\alpha}}(k+d)$. We use the margin maximisation algorithm (MMA). The term margin refers to the excess power level applied to a transmitted symbol so that the receiver SNR exceeds the minimum threshold required to achieve the target error probability $\mathcal{P}_{e}$, subject to the selected symbol rate. The MMA scheme was implemented for this task as it seeks to distribute power across subchannels in such a way as to best equalise outage rates across sub-carriers, sublect to available power constraints.

We also investigate the predictive allocation power penalty, defined as the excess total transmitter power required to achieve a given value of symbol outage probability $\mathcal{P}_{o}$ for the particular prediction range under test. Each subchannel is assumed to have a $3 \mathrm{kHz}$ bandwidth and it is assumed that the frequency response is flat within each individual narrowband subchannel.

We evaluate the receiver SNR obtained after the MMA has been performed and after attenuation by the transmission channel. This is compared with the SNR levels required to satisfy the target selected rate on each subchannel. We thus detect whether an outage occurred during each interval and hence determine the overall outage rate. More details of this evaluation are presented in Table I.

Table I: Details of Adaptive Allocation for Penalty Analysis

\begin{tabular}{|l|c|}
\hline No. of Subcarriers & 6 \\
\hline Subchannel Bandwidth & $3 \mathrm{kHz}$ \\
\hline Modulation Scheme & M-QAM \\
\hline Maximum Modulation Depth & $6 \mathrm{bits}$ \\
\hline Target Bit Error Probability & $10^{-2}$ \\
\hline Prediction Filter & $\mathrm{LMS}$ \\
\hline No. of Filter Taps & 2 \\
\hline Step Size, $\mu$ & 0.1 \\
\hline Allocation Algorithm & $\mathrm{MMA}$ \\
\hline Noise PSD, $N_{o}$ & $10^{-9} \mathrm{~W} / \mathrm{Hz}$ \\
\hline Source Data Scaling & $10^{4}$ \\
\hline \hline
\end{tabular}

\section{RESUlts}

\section{A. CSI Prediction}

We examined the relationship between predictor MSE and prediction range for these data and present the results in Fig. 5. A $4^{\text {th }}$ order best-fit polynomial curve for these data points is 
also included in the diagram. These results indicate that the filter MSE increases with an increase in prediction range and also that the error is small as compared to the filter gain, even for a considerable prediction range.

The prediction shortfall probability performance was also evaluated as shown in Fig. 6: the best-fit curve is also a $4^{\text {th }}$ order approximation. Fractional shortfall probability was also assessed by comparing the predicted CSI value with fractions of the actual CSI obtained. This shortfall probability is observed to be higher at low prediction ranges and decreases with increasing prediction range. These results indicate that CSI prediction is feasible in an actual wireless transmission environment. In Fig. 7, we present sample prediction results obtained on a single subchannel for $\mu=0.1, F=2$ and various values of $d$. We also note the applicability of singlestep prediction to current OFDM systems: the $109 \mathrm{~ms}$ channel sample interval is large compared to typical symbol duration.

\section{B. Resource Allocation}

Typical resource allocation results obtained for the application of the MMA on the 6-subchannel system are presented in Fig. 8 .

We also investigated the power penalty associated with applying the MMA to the predicted CSI and present the results of this operation in Fig. 9. It may be seen that an increase of the transmitter power is associated with a decreasing outage probability $\mathcal{P}_{o}$. For these data, we also observe a small increase in $\mathcal{P}_{o}$ as the prediction horizon is increased.

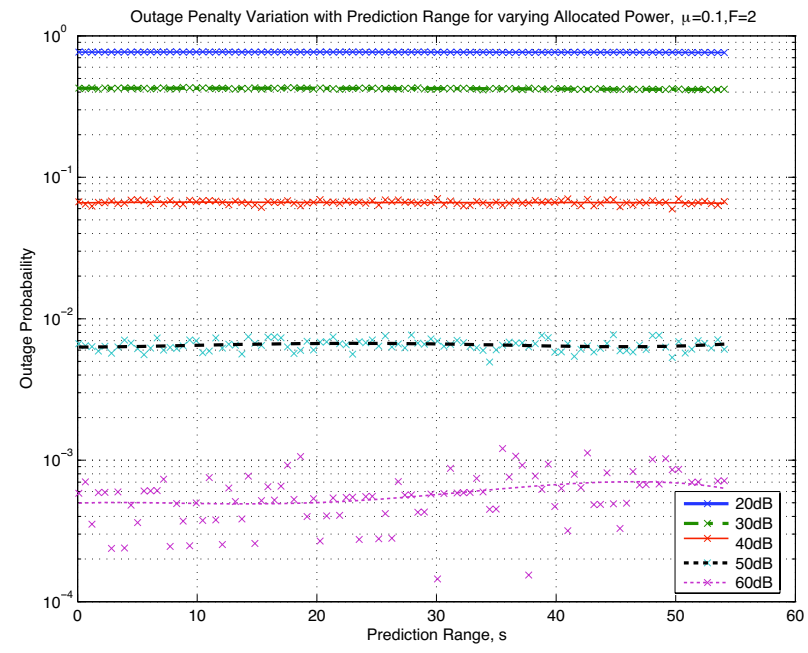

Figure 9: Variation of Long-Range Predictive Allocation Penalty with Prediction Depth

\section{CONCLUSION}

These results gleaned from these analyses of suburban mobile wireless transmission environments motivate the recommendation of predictive resource allocation in realistic transmission scenarios. Following [13], we note that if a bisection search is performed in determining the optimal ratepower distribution, then each iteration of the algorithm will require at most $2(N-1)$ additions and $N$ multiplications, or at worst 763 flops of a computer. Additionally, the algorithm provided an optimal distribution for a 255-subcarrier OFDM transmission within 11 iterations ( $\leq 8,400$ flops). If a $1 \mathrm{MHz}$ speed processor is used to perform this evaluation, then the use of this algorithm will provide optimal RP within $8.4 \mathrm{~ms}$. Furthermore, increasing the CSI prediction range will require correspondingly less computing resources for transmission resource optimisation but will not incur an onerous cost in terms of predictor shortfall probability.

Additional research will therefore enable the system designer to select a desired operating point in terms of transmission power constraints, available processing power, desired outage behaviour and target data throughput.

\section{ACKNOWLEDGMENT}

The authors would like to thank Prof. Anders Ahlen of Uppsala University, Sweden; Dr. Paul D. Teal of Industrial Research Limited, New Zealand; and also Drs. Sören Andersson and Henrik Asplund of Ericsson Radio Systems AB, Sweden for allowing access to the original channel fading sample data forming the basis for this work.

\section{REFERENCES}

[1] B. S. Krongold, K. Ramchandran, and D. L. Jones, "Computationally efficient optimal power allocation algorithm for multicarrier communication systems," IEEE Transactions on Communications, vol. 48, no. 1, pp. 23-27, January 2000.

[2] H. Asplund, A. A. Glazunov, and J.-E. Berg, "An investigation of measured and simulated wideband channels with applications to 1.25 MHz and 5 MHz cdma systems," Proc. 48th IEEE Vehicular Technology Conference, vol. 1, pp. 562-566, May 1998.

[3] M. Sternad and D. Aronsson, "Channel estimation and prediction for adaptive ofdma/tdma uplinks, based on overlapping pilots," Proceedings IEEE International Conference on Acoustics, Speech, and Signal Processing (ICASSP '05), vol. 3, pp. iii/861 - iii/864, March 2005.

[4] S. Gifford, C. Bergstrom, and S. Chuprun, "Adaptive and linear prediction channel tracking algorithms for mobile ofdm-mimo applications," IEEE Military Communications Conference, 2005. MILCOM 2005., pp. $1-5$, October 2005.

[5] D. Schafhuber and G. Matz, "Mmse and adaptive prediction of timevarying channels for ofdm systems," IEEE Transactions on Wireless Communications, vol. 4, no. 2, pp. 593 - 602, March 2005.

[6] M. Münster and L. Hanzo, "Mmse channel prediction assisted symbolby-symbol adaptive ofdm," IEEE International Conference on Communications, 2002, vol. 1, pp. 416 - 420, April 2002.

[7] S. Falahati, A. Svensson, M. Sternad, and T. Ekman, "Adaptive modulation systems for predicted wireless channels," Proc. IEEE Global Conference on Communications GLOBECOM, pp. 357-361, 2003.

[8] K. Prince, B. S. Krongold, and S. Dey, "Prediction-based resource allocation for OFDM in wireless channels," Proceedings of the 2005 Australia Communications Theory Workshop, Brisbane, Australia, pp. 241-245, February 2005.

[9] — , "A framework for efficient rate-power allocation for OFDM in a composite-fading environment," Proceedings of the IEEE International Conference on Communications (ICC), Seoul, South Korea, vol. 4, pp. 2558-2562, May 2005.

[10] - "OFDM transmission resource optimization in HMM-fading wireless channels," Proc. International Association of Science \& Technology for Development, Banff, Alberta, Canada, July 2005.

[11] S. M. Kay, Fundamentals of Statistical Signal Processing: Esimation Theory. Upper Saddle River, NJ 07458: Prentice Hall PTR, 1993.

[12] E. Sousa, V. Jovanovic, and C. Daigneault, "Delay spread measurements for the digital cellular channel in toronto," IEEE Transactions on Vehicular Technology, vol. 43, no. 4, pp. 837-847, November 1994.

[13] B. S. Krongold, "New techniques for multicarrier communication systems," Ph.D. dissertation, University of Illinois at Urbana-Champaign, 2001. 